Ophthalmologe 2020 $\cdot 117: 497-498$ https://doi.org/10.1007/s00347-020-01052-2 Online publiziert: 30 . Januar 2020 (c) Springer Medizin Verlag GmbH, ein Teil von Springer Nature 2020

\title{
Vinodh Kakkassery
}

Klinik für Augenheilkunde, Universitätsklinikum Schleswig-Holstein, Campus Lübeck Universität zu Lübeck, Lübeck, Deutschland

\section{Personalisierte Medizin in der Behandlung von Augentumoren}

\section{Verbindung zwischen Molekularbiologie, Klinik und den persönlichen Bedürfnissen des Patienten}

Neue Veröffentlichungen und ein Zugewinn an Informationen zum Thema Tumor sind nahezu täglich zu konstatieren. Wöchentliche Listungen von Veröffentlichungen in medizinischen Suchmaschinen wie Medline verdeutlichen, dass sich auch in der Augentumorbehandlung das evidenzbasierte Wissen massiv erweitert. Gerade vor diesem Hintergrund, aber auch mit der Abkehr von strikt vorgegebenen Behandlungspfaden für Krankheiten in der Medizin befreien sich Arzt und Patient ein wenig aus den bisherigen engen Denkstrukturen. Die Nutzung von pharmakogenetischen Aspekten, bei denen spezifische Mutationsänderungen im Tumorgewebe Ansätze für eine Therapie ergeben, sind hier ein einfach verständliches Beispiel und der bekannteste Ansatz der personalisierten Medizin. Allerdings sind weitere Differenzierungsmöglichkeiten von Tumoren in der Diagnostik auf Protein- oder Mediatorenebene von besonderer Bedeutung und weisen auf unterschiedliche Therapiestrategien als „best care“ abhängig vom vorliegenden Fall hin.

Neben der molekularbiologischen und genetischen Individualisierung einer Augentumorerkrankung sollen und werden andere Aspekte wie Klinik und Komorbiditäten, aber auch die persönliche Lebenssituation und Wünsche eines Patienten in die Waagschale zur Therapieentscheidung geworfen. Während Klinik und Komorbiditäten z. B. zur Limitation einer körperlich belastenden zielgerichteten Systemtherapie führen können, sind persönliche Lebenssituationen, wie z. B. eine fehlende Versorgung des Patienten zu Hause, eine Schwangerschaft oder auch die fehlende Möglichkeit, die ambulanten Therapietermine wahrzunehmen, Kriterien für eine Therapieauswahl.

》) Bei der Therapieentschei-
dung werden Lebenssituation
und Wünsche des Patienten
berücksichtigt

Letztendlich ist der Paradigmenwechsel von einer Algorithmen-gesteuerten Behandlung $\mathrm{zu}$ einer vom Patienten ausgewählten Therapie die vielleicht größte Veränderung zu vorherigen Behandlungsstrategien. Das onkologische Team, zu dem auch der augentumorerfahrene Augenarzt gehört, bietet nach dem Diskurs der Patientensituation in einer Tumorkonferenz ein Behandlungskonzept an. Dieses orientiert sich an den molekularbiologischen und -genetischen Charakteristika des Tumors zum einem, aber zum anderen auch an der Klinik, an der individuellen Lebenssituation und an den Wünschen des Patienten. Die Vorteile bei der Berücksichtigung der Wünsche des Patienten liegen auf der Hand. Der Patient ist Subjekt in dem Prozess der Behandlung und braucht eine Therapie nicht nur „über sich ergehen zu lassen“. Ebenso kann die positive Haltung des Patienten gegenüber der ausgewählten 
Therapie genutzt werden und so dem Behandlungsprozess zugutekommen. Arzt und Patient kommen sich in diesem individuellen und weniger mechanischen Ansatz der Therapieauswahl deutlich näher.

In diesem Sinne sind exemplarisch 3 Bereiche aus der Augentumortherapie in diesem Heft aufgegriffen und im Hinblick auf die personalisierte Medizin neu beleuchtet worden. Mit den Aderhautmelanomen, den okulären Lymphomen sowie den Tumoren im Lidbereich und auf der Augenoberfläche kann in diesem Heft durchaus gelungen dargestellt werden, inwieweit die personalisierte Medizin in der Behandlung der Augentumoren bereits greift und wie dieser Ansatz weiter vertieft werden kann und sollte [1-3].

Ihr

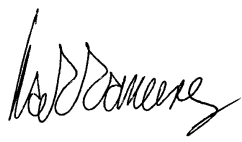

PD. Dr. Vinodh Kakkassery

\section{Korrespondenzadresse}

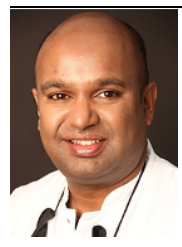

PD. Dr. med.

\section{Vinodh Kakkassery}

Klinik für Augenheilkunde,

Universitätsklinikum

Schleswig-Holstein, Campus

Lübeck Universität zu Lübeck

Ratzeburger Allee 160,

23538 Lübeck, Deutschland vinodh.kakkassery@ gmail.com

Interessenkonflikt. V. Kakkassery gibt an, dass kein Interessenkonflikt besteht.

\section{Literatur}

1. Kakkassery $V$, Jünemann $A M$, Bechrakis NE et al (2019) Lymphom am Auge. Ophthalmologe. https://doi.org/10.1007/s00347-019-01020-5

2. Grisanti S, Ranjbar M, Tura A et al (2019) Uveales Melanom. Ophthalmologe. https://doi.org/10. 1007/s00347-019-01001-8

3. Rokohl AC, Koch KR, Mor JM et al (2020) Personalisierte Medizin in der Behandlung von periokulären Tumoren. Ophthalmologe. https:// doi.org/10.1007/s00347-019-01034-z

\section{Neuerscheinungen in der Augenheilkunde}

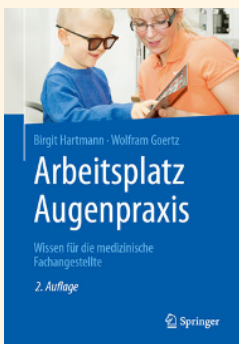

Arbeitsplatz Augenpraxis.

Wissen für die medizinische Fachangestellte

Dr. Birgit Hartmann, Dr. Wolfram Goertz

2. Aufl. 2019, 221 S., Softcover, $44,99 €$

ISBN 978-3-662-59105-5

Was wäre der Augenarzt ohne sein Praxisteam! Das Buch gibt

Antworten auf alle wichtigen Fragen, die medizinische Fachangestellte in der Augenheilkunde beantworten können müssen.

- Wie kann man sich für die Arbeit an der Anmeldung rüsten?

- Was tun bei Patienten mit Glaukomanfall, wenn jede Sekunde zählt?

- Wie wird die Sehschärfe geprüft?

- Was wird bei der Augenuntersuchung genau untersucht?

- Wie wird der Abfluss beim Tränenwegverschluss wiederhergestellt?

- Was ist für die Abrechnung wichtig?

Da der Bereich Augenheilkunde in der Ausbildung nur gestreift wird, müssen Auszubildende und Medizinische Fachangestellte, die in einer Augenpraxis oder -klinik arbeiten möchten, ihr Wissen durch "learning by doing" und mit Hilfe von Weiterbildungen erwerben. Ihr Praxisteam profitiert vom Erfahrungsschatz des renommierten Autorenteams und Ihre Angestellten gewinnen schnell die gewünschte Sicherheit am "Arbeitsplatz Augenpraxis".

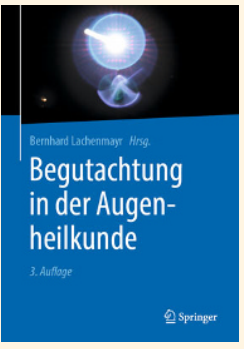

Begutachtung in der Augenheilkunde

Prof. Dr. Dr. Bernhard Lachenmayr

3. Aufl. 2019, 300 S., Hardcover, 89,99€

ISBN 978-3-662-58922-9

Das Buch vermittelt fundiert und praxisnah die korrekte Durchführung und Dokumentation einer augenärztlichen Begutachtung und die aktuellen juristischen Grundlagen. Anhand zahlreicher Fallbeispiele, Mustergutachten, Tipps und Abbildungen erhält der Arzt detaillierte Handlungsanweisungen für die Begutachtung. Alle Inhalte sind auf die anerkannten Richtlinien der Fachgesellschaften abgestimmt und sorgen so für Rechtssicherheit. In der aktualisierten 3. Auflage finden Sie alle relevanten Rechts- und Versicherungsbereiche mit den aktuell gültigen Verordnungen, u.a. Eignung für Straßen-, Flug-, Schiffs- und Bahnverkehr, Berufliche Eignung, Begutachtung für die Gesetzliche Unfallversicherung und für die Private Unfallversicherung, Blindheitsbegutachtung.

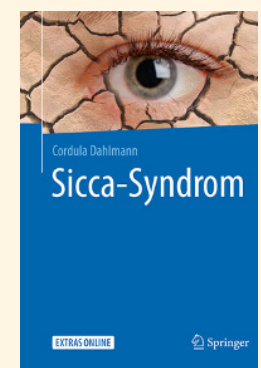

\section{Sicca-Syndrom}

Cordula Dahlmann

1. Aufl. 2019, 139 S., Softcover, $29,99 €$, ISBN 978-3-662-56408-0

Das Buch beleuchtet in prägnanter Form das Krankheitsbild der Keratokonjunktivitis sicca, welches immer mehr in den Fokus des Praxisalltags rückt. Die Ursachen für die Beschwerden des trockenen Auges sind komplex, der Leidensdruck der Betroffenen hoch. Hier setzt dieses Buch an: In kompakter, stichwortartiger Form bietet es eine praktische Orientierung zum diagnostischen und therapeutischen Management. Die klinischen Ursachen werden strukturiert beschrieben. Ein Vorschlag zur praktischen Testreihenfolge sowie 12 praxisnahe Falldarstellungen illustrieren die zielgerichtete Beratung und Therapie der Betroffenen. Eine Excel-Tabelle zum Download bietet einen Überblick über die gängigen Tränenersatzmittel und deren Eigenschaften und hilft damit, eine optimale Basistherapie für die unterschiedlichen Patienten zu finden.Ergänzende Methoden wie Akupunktur sowie psychosomatische Aspekte bieten erweiterte Methoden im therapeutischen Spektrum. Damit stellt dieses Buch eine praktische und übersichtliche Hilfe für Ärzte, Apotheker und Optiker zur optimalen Betreuung ihrer Patienten bzw. Kunden mit Sicca-Syndrom dar. 